\title{
Malformación congénita de la vía aérea pulmonar o malformación adenomatoidea quística congénita. Serie clínica de 16 pacientes
}

\author{
GUILLERMO ZEPEDA F.*, MARGARITA ALDUNATE R.**, VERÓNICA RUIZ D.***, \\ RODRIGO BOZZO H.**** y LUIS VELOZO P.*****
}

\section{Congenital pulmonary airway malformation or congenital cystic adenomatoid malformation}

Objective: To describe 16 patients treated for a congenital pulmonary anomaly named congenital pulmonary airway malformation (CPAM) or congenital cystic adenomatoid malformation (CCAM) in Roberto del Río children's Hospital. Methods: We retrospectively analyzed the medical records of patienes who had had surgery for CPAM/CCAM from January 2000 to July 2014. Results: We found 16 cases, 9 of them were males. Nine had surgery within the first year of life (5 of them within 3 weeks of life). According to Stocker's classification CPAM/CCAM was type 1 in 9 patients, and type 2 in 7. Diagnosis was based on antenatal ultrasonography $s(n=10)$, persistent abnormalities in Chest X-Ray $(n=4)$, recurrent pneumonia $(n=1)$ and incidental radiological findings $(n=1)$. Computed Tomography $(C T)$ was performed in 13 patients, CT angiography in 2 and only chest $x$-ray in one. The lesions were located in right upper lobe (3), right middle lobe (2), right lower lobe (4), left upper lobe (4) and left lower lobe (3). One patient had pectus excavatum and another one had ventricuar septal defect. They all had open thoracotomy with lobectomy of the affected area. Postoperatively was uneventful except for transient lobar atelectasis in 2 patients. There was no mortality. Conclusions: We emphasize the early diagnosis and good outcome of our patients treated which this disease. Our findings are consistent with those that has been reported in the literature.

Key words: Congenital pulmonary airway malformation, congenital cystic adenomatoid malformation, congenital lung malformations, congenital thoracic malformation.

\section{Resumen}

Objetivo: Describir los pacientes tratados por malformación congénita de la vía aérea pulmonar (MCVAP) o malformación adenomatoidea quística congénita (MAQC) en el Hospital de niños Roberto del Río desde el año 2000 hasta el segundo semestre de 2014. Métodos: Revisión retrospectiva de fichas clínicas. Resultados: 16 pacientes, 9 hombres. Se operaron antes del año de vida 9 pacientes (5 de ellos antes de las 3 semanas de vida). Tipo de MCVAP (según clasificación de Stocker) tipo 1 fue 9/16 y tipo 2 fueron 7/16. La sospecha diagnóstica fue en base a diagnóstico prenatal 10/16, imagen radiológica persistente 4/16, neumonías repetidas 1/16 y hallazgo radiológico 1/16. Estudio imagenológico: Tomografia computada (TC) 13/16, Angio-TC 2/16 y radiografia de tórax 1/16. Ubicación: lóbulo superior derecho $3 / 16$, lóbulo medio 2/16, lóbulo inferior derecho 4/16, lóbulo superior izquierdo 4/16, lóbulo inferior izquierdo 3/16. Comorbilidad: ninguna 14/16, pectus excavatum 1/16, comunicación

\footnotetext{
* Broncopulmonar Infantil. Magister en Educación en Ciencias de la Salud. Profesor Asistente Departamento de Pediatría y Cirugía Infantil, Campus Norte, Facultad de Medicina Universidad de Chile. Hospital Roberto del Río.

** Cirujana infantil. Departamento de Pediatría y Cirugía Infantil, Campus Norte, Facultad de Medicina Universidad de Chile. Hospital Roberto del Río.

*** Becaria de Programa de Especialista en Pediatría. Departamento de Pediatría y Cirugía Infantil, Campus Norte, Facultad de Medicina Universidad de Chile. Hospital Roberto del Río.

**** Broncopulmonar infantil. Hospital Roberto del Río.

*****Anatomopatólogo. Hospital Roberto del Río.
} 
interventricular 1/16. Tratamiento quirúrgico: en todos los pacientes se realizó lobectomía total del lóbulo afectado por vía abierta. Evolución postoperatoria: Buena 14/16, atelectasia 2/16. No se registró mortalidad. Conclusiones: Se destaca el diagnóstico precoz y buena evolución de nuestros pacientes concordantes con la literatura.

Palabras clave: Malformación congénita de la vía aérea pulmonar, malformación adenomatoidea quística congénita, malformación pulmonar congénita, malformación torácica congénita.

\section{Introducción}

La malformación congénita de la vía aérea pulmonar (MCVAP), terminología instaurada por Stocker ${ }^{1}$ o malformación adenomatoidea quística congénita (MAQC), terminología utilizada por la European Respiratory Society ${ }^{2}$, es una infrecuente malformación pulmonar con una prevalencia en 0,81 por 10.000 recién nacidos vivos según el registro epidemiológico europeo de malformaciones congénitas ${ }^{3}$. Para efectos prácticos, en este artículo nos referiremos a esta patología como MCAVP/MAQC.

Según Stocker, se clasifica en los tipos 0 a 4 siguiendo una hipótesis sobre su patogenia progresiva en que el tipo 0 es un defecto esencialmente traqueobronquial hasta el tipo 4 que es un defecto alveolar ${ }^{1,4}$. Langston por su parte, propone otra clasificación basándose en el tamaño de los quistes con o sin conexión vascular, tipo de hiperplasia pulmonar y patología tumoral ${ }^{5}$. Estas dos clasificaciones son las más utilizadas y este trabajo utilizará la clasificación de Stocker para referirse a los tipos de MCVAP.

La sintomatología causada por la MCVAP/ MAQC es muy variada dependiendo principalmente del tamaño de la malformación, ubicación, edad del paciente, asociación con otras malformaciones y complicaciones de la misma ${ }^{2,6-13}$.

En Chile no existen series clínicas recientes que describan esta patología, sólo se describe una publicación en la década de los noventa con una serie de $6 \operatorname{casos}^{14}$ y reportes recientes de casos aislados de presentación adulta ${ }^{15,16}$ por lo que el objetivo de este trabajo es presentar la experiencia acumulada de MCVAP/MAQC en el Hospital de niños Roberto del Río en los últimos años de manera de contribuir al mejor entendimiento de esta patología.

\section{Metodología}

Se presenta un estudio de tipo retrospectivo y descriptivo en el cual se realizó una búsqueda en la base de datos computacional del Servicio de Anatomía Patológica del Hospital Roberto del Río de los pacientes operados con diagnóstico anatomopatológico de MCVAP/MAQC registrados desde enero de 2000 hasta julio de 2014. Luego se obtuvieron los datos desde las fichas clínicas en sus aspectos demográficos (edad, sexo), clínicos (forma de presentación, presencia de comorbilidad), radiológicos (tipo de imagen, resultado de imagen, ubicación de MCVAP/ MAQC), quirúrgicos (tipo de cirugía, evolución, complicaciones quirúrgicas, estadía hospitalaria) y anatomopatológicos (informe de biopsia) y se llevaron a planilla de cálculo Excel para el análisis respectivo. Los datos son expresados como valores absolutos, porcentajes o mediana.

\section{Resultados}

Se registraron 16 pacientes en el período siendo $9(56 \%)$ de género masculino. Los tipos de MCVAP/MAQC fueron nueve de tipo 1 (56\%), siete tipo $2(44 \%)$, no se registraron los tipos 0,3 ni tipo 4. El análisis indicó una edad de diagnóstico $\mathrm{y} / \mathrm{u}$ operación en que predominaron los menores de un año, especialmente los recién nacidos, con 9 y 5 pacientes respectivamente. Se destaca que los menores de un mes fueron 5 pacientes, siendo sus edades $2,7,9,15$ y 18 días de vida (Tabla 1).

La ubicación según el lóbulo comprometido no indicó un claro predominio de ninguno, ya que,

Tabla 1. Características generales de 16 pacientes operados de malformación congénita de la vía aérea pulmonar (MCVAP/MAQC)

\begin{tabular}{|c|c|c|}
\hline \multicolumn{3}{|l|}{ Género } \\
\hline \multicolumn{3}{|c|}{ Edad al efectuar la cirugía } \\
\hline Menores de 1 año & $\mathrm{n}(\%)^{*}$ & $9(56)$ \\
\hline 1 a 5 años & $\mathrm{n}(\%)$ & $3(19)$ \\
\hline 6 a 10 años & $\mathrm{n}(\%)$ & $3(19)$ \\
\hline 10 a 14 años & $\mathrm{n}(\%)$ & $1(6)$ \\
\hline \multicolumn{3}{|c|}{ Tipo de malformación según Stocker ${ }^{1}$} \\
\hline Tipo 1 & $\mathrm{n}(\%)$ & $9(56)$ \\
\hline Tipo 2 & $\mathrm{n}(\%)$ & $7(44)$ \\
\hline
\end{tabular}

* Se destaca que los menores de 1 mes fueron 5 pacientes, siendo sus edades $2,7,9,15$ y 18 días de vida. 
Tabla 2. Ubicación de tipos de malformación congénita de la vía aérea pulmonar (MACVAP/MACQ) según lóbulo afectado en 16 pacientes

\begin{tabular}{|llccc|}
\hline & & MCVAP/MACQ tipo 1 & MCVAP/MACQ tipo 2 & Total n (\%) \\
\hline LSD & (n) & 3 & 0 & $3(19)$ \\
LM & (n) & 2 & 0 & $2(12)$ \\
LID & (n) & 1 & 3 & $4(25)$ \\
LSI & $(\mathrm{n})$ & 3 & 1 & $4(25)$ \\
LII & (n) & 0 & 3 & $3(19)$ \\
Total & $\mathrm{n}(\%)$ & $9(56)$ & $7(44)$ & $16(100)$ \\
\hline
\end{tabular}

LSD: lóbulo superior derecho. LM: lóbulo medio. LID: lóbulo inferior derecho. LSI: lóbulo superior izquierdo. LII: lóbulo inferior izquierdo.

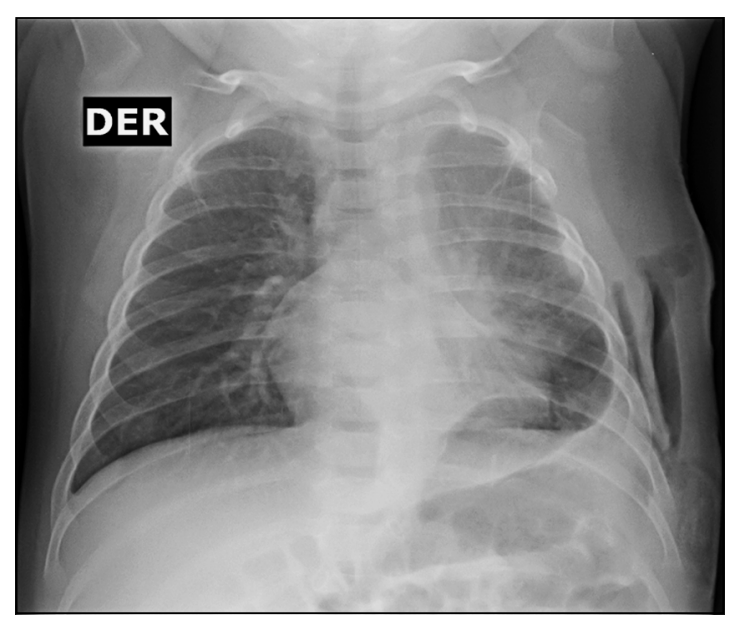

Figura 1. Radiografía de tórax de paciente de 7 meses con malformación congénita de la vía aérea pulmonar tipo 2 de lóbulo inferior izquierdo.

los lóbulos superiores se afectaron en 7 pacientes $(44 \%)$, los lóbulos inferiores en 7 pacientes (44\%) y el lóbulo medio en 2 pacientes (12\%). La ubicación de las MCVAP/MAQC tipo 1 comprometió a 3 pacientes en los lóbulos superiores tanto derecho como izquierdo, a 2 pacientes en el lóbulo medio y a un paciente en el lóbulo inferior derecho, mientras que las tipo 2 afectaron a los lóbulos superior izquierdo, inferior izquierdo e inferior derecho con 1,3 y 3 pacientes respectivamente (Tabla 2).

El diagnóstico imagenológico se logró con tomografía computada (TC) en 13 pacientes, con Angio-TC en 2 pacientes y en un paciente se sospechó el diagnóstico con radiografía de tórax (Figuras 1 a 3).

En el período neonatal 5 pacientes fueron asintomáticos y uno presentó distrés respiratorio

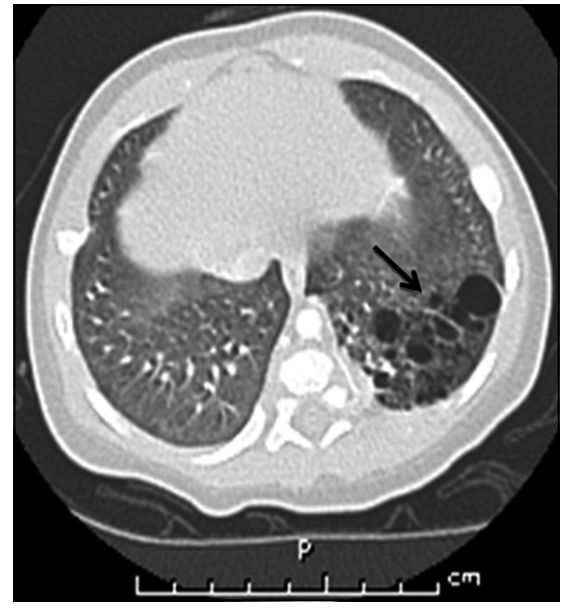

Figura 2. Tomografía computada de un paciente portador de MACVAP/MACQ tipo 2 del lóbulo inferior izquierdo (flecha negra) (es el mismo paciente de la Figura 1).

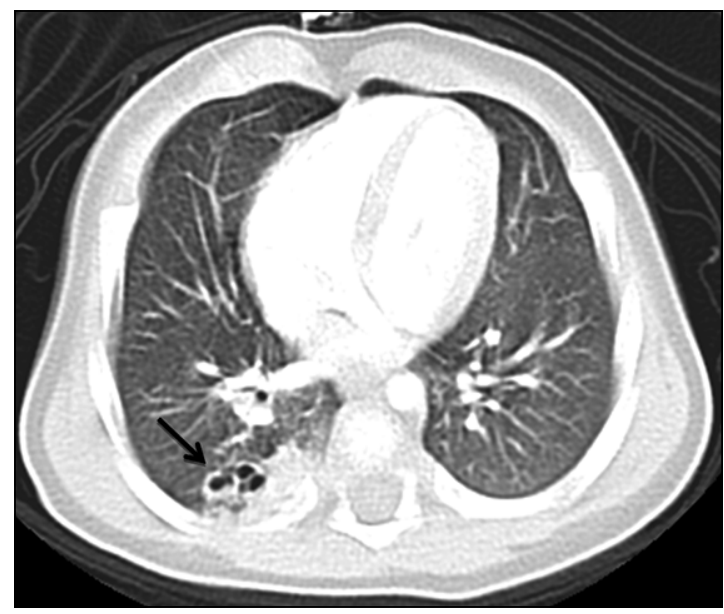

Figura 3. Tomografía computada de un paciente portador de MACVAP/MACQ tipo 2 de lóbulo inferior derecho (flecha negra) operado a los 7 meses. 
Tabla 3. Orientación diagnóstica de los tipos de malformación congénita de la vía aérea pulmonar (MACVAP/MACQ) en 16 pacientes

\begin{tabular}{|c|c|c|c|}
\hline & MCVAP/MACQ tipo 1 & MCVAP/MACVAP tipo 2 & Total n (\%) \\
\hline Diagnóstico prenatal (n) & 5 & 5 & $10 \quad(63)$ \\
\hline Imagen persistente $\quad(n)$ & 3 & 1 & $4(25)$ \\
\hline Neumonía recurrente (n) & 1 & 0 & $1 \quad(6)$ \\
\hline Hallazgo radiológico (n) & 0 & 1 & $1 \quad(6)$ \\
\hline Total & 9 & 7 & $16(100)$ \\
\hline
\end{tabular}

severo. En el período de lactante menor de 1 año dos pacientes evolucionaron asintomáticos y uno con infección respiratoria baja repetida. En el período desde el año hasta los 5 años dos pacientes evolucionaron asintomáticos (tenían diagnóstico prenatal) y uno con infecciones respiratorias y obstrucciones bronquiales bajas repetidas. Un

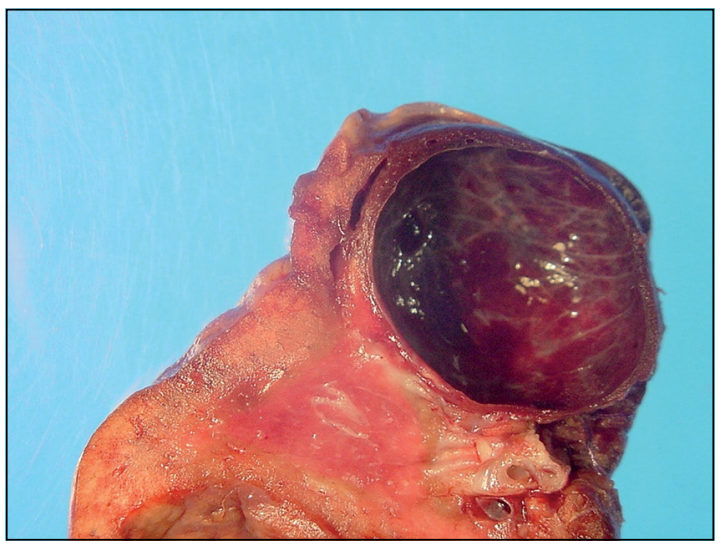

Figura 4. Malformación congénita de la vía aérea pulmonar (MACVAP/MACQ) tipo 1. Se aprecia lóbulo con un gran quiste extraído de un paciente escolar.

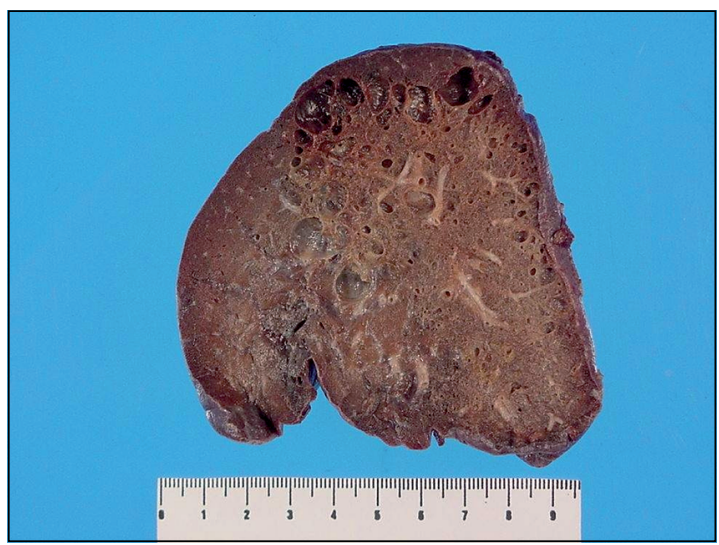

Figura 5. Malformación congénita de la vía aérea pulmonar (MACVAP/MACQ) tipo 2. Se aprecia malformación con numerosos quistes de menos de $2 \mathrm{~cm}$ de diámetro en $\mathrm{su}$ interior dando un aspecto esponjoso. paciente escolar tenía el antecedente anamnéstico de neumonía repetida (no se realizó radiografía de tórax entre episodios) dos pacientes escolares presentaron infección respiratoria baja repetida y finalmente, la serie incluyó a un paciente adolescente asintomático, en quien el diagnóstico fue hecho por un hallazgo radiológico.

La sospecha diagnóstica indicó un predominio del diagnóstico prenatal en ambos tipos de MCVAP/MAQC con un total de 10 pacientes. La imagen radiológica persistente se evidenció en 4 pacientes. La neumonía repetida apareció como dato anamnéstico entregado por los padres de una paciente procedente del extranjero que vino a Chile en busca de una solución a su patología. El diagnóstico se hizo por hallazgo radiológico en un paciente estudiado por causa extrapulmonar, que era asintomático hasta ese momento (Tabla 3).

En dos pacientes se detectó comorbilidad: un paciente presentó pectus excavatum con MCVAP tipo $1 \mathrm{y}$ otro paciente que presentaba una comunicación interventricular era portador de MCVAP tipo 2.

El tipo de cirugía elegida en todos los pacientes fue la toracotomía abierta con lobectomía del sitio afectado (Figuras 4 y 5).

La evolución de los pacientes fue satisfactoria, registrándose ausencia de complicaciones en 14 pacientes. Sólo 2 pacientes presentaron atelectasias lobares en el postoperatorio que se resolvieron con kinesiterapia respiratoria, en un plazo no mayor a 2 días. No se registró mortalidad (Tabla 4).

Tabla 4. Evolución de los 16 pacientes operados de malformación congénita de la vía aérea pulmonar (MACVAP/MACQ)

\begin{tabular}{|lc|}
\hline Evolución & \\
\hline Buena & $14(87,5 \%)$ \\
\hline Atelectasia & $2(12,5 \%)$ \\
\hline Mortalidad & 0 \\
\hline
\end{tabular}


La mediana de la estadía hospitalaria postcirugía correspondió a 5,5 días con un rango de 4 a 8 días. Se excluyó de este análisis a los recién nacidos (en total 5) debido a la dificultad para realizar un análisis sólo del acto operatorio en virtud de patologías neonatales médicas, sin embargo, el alta quirúrgica fue tan precoz como $48 \mathrm{~h}$.

\section{Discusión}

La MCVAP/MAQC es una de las malformaciones pulmonares que es factible detectar en la vida intrauterina y que puede ocasionar diferentes complicaciones a lo largo de la vida como infecciones respiratorias repetidas, bronquiectasias, abscesos pulmonares, hemoptisis, neumotórax, pioneumotórax, y más raramente transformación maligna $^{2,6}$. Según Stocker, las MCVAP/MAQC se dividen en el tipo 0 que corresponde a la displasia acinar traqueobronquial cuyo origen del defecto en la vida intrauterina sería traqueobronquial y es incompatible con la vida. En el tipo 1 el sitio de la alteración en la vida intrauterina estaría en la región bronquial/bronquiolar y presenta quistes de hasta $10 \mathrm{~cm}$ de diámetro presentando epitelio de tipo pseudoestratificado ciliado intercalado por células mucosas. En el tipo 2 el origen del defecto estaría en la región bronquiolar y es de tipo esponjoso con múltiples quistes hasta $2 \mathrm{~cm}$ de diámetro y tejido pálido sólido presentando epitelio de tipo cilíndrico ciliado sin evidencias de cartílago y el músculo estriado puede estar presente en 5\%. En el tipo 3 el defecto estaría en la región bronquiolar/alveolar y es de tipo sólido con epitelio de tipo cúbico sin tejido pulmonar entre los quistes. En el tipo 4 el origen de la alteración estaría en la región acinar distal y son quistes periféricos de pared delgada, de pared alveolar y se consideran blastomas pleuropulmonares tipo 1 que involucionaron en su histología ${ }^{1,4}$.

La serie clínica presentada, aunque pequeña, nos indica diversos hechos relevantes. En nuestros pacientes no hubo un claro predominio de género tal como lo relatan algunas series clínicas ${ }^{10}$ y se confirma al MCVAP /MAQC tipo 1 como la más frecuente seguida de la MCVAP tipo 2 como se ha publicado anteriormente tanto a nivel nacional ${ }^{14}{ }^{\text {como internacional }}{ }^{10,11}$. El hecho de no encontrar en nuestra serie casos como los tipo 0,3 y 4 es propio de series con una bajo número de pacientes ${ }^{11}$.

Destacamos en nuestra serie la precocidad en el diagnóstico. Nuestros pacientes con diagnóstico prenatal y/o neonatal precoz fueron operados en forma temprana como se ha sugerido en la literatura, con la finalidad de evitar complicaciones como distrés respiratorio en el período neonatal o en etapas posteriores infecciones $\mathrm{y} / \mathrm{o}$ neoplasias ${ }^{2,6}$. Existe consenso en la literatura en que los recién nacidos que presentan distrés respiratorio atribuible a esta patología se deben operar en cuanto las condiciones lo permitan ${ }^{2}$. Por otra parte, no existe consenso en el tiempo operatorio de niños asintomáticos, se ha sugerido que es posible realizar una cirugía segura en niños entre los 9 y 12 meses de vida ${ }^{6}$.

En relación a la ubicación, nuestros resultados indican un leve predominio derecho ya publicado en otras series que describen un $72 \%$ de lateralidad y no se observó un predominio de algún lóbulo en particular tal como se ha señalado en otras series ${ }^{10}$.

La sospecha diagnóstica predominante fue el diagnóstico prenatal en un $63 \%$. Una serie nacional de 6 pacientes comunica un diagnóstico prenatal en 4 pacientes ${ }^{14}$. Estos valores difieren a lo descrito en la literatura de países desarrollados que sugieren como óptimos porcentajes de $90 \%{ }^{17}$, pero también difieren de series latinoamericanas que describen al diagnóstico prenatal con valores de $11 \%{ }^{10}$. En relación a este punto, se ha destacado la importancia de la acuciosidad de la ultrasonografía (US) obstétrica y su disponibilidad para el total de la población materna. Es el método de elección para realizar el diagnóstico en forma prenatal y en algunos casos específicos puede ser complementada con Resonancia Magnética fetal $^{18,19}$.

La segunda sospecha diagnóstica con un $25 \%$ fue la imagen radiológica persistente. En este punto es importante la sospecha diagnóstica cuando existan lesiones pulmonares que no mejoren después de una consolidación parenquimatosa considerada como neumonía, ya que, las MCVAP/MAQC se han descrito como causa de neumonía recurrente en la misma área del pulmón ${ }^{20}$. Un paciente proveniente del extranjero se estudió en nuestro centro entregándose al ingreso el dato anamnéstico de neumonía recurrente y al realizar el estudio correspondiente se llegó al diagnóstico, en este punto cobra importancia realizar radiografías de control cuando se considere tratada una neumonía para de esta manera lograr un diagnóstico correcto.

Los estudios de imágenes son fundamentales para lograr un diagnóstico preoperatorio óptimo y para descartar la presencia de complicaciones o anomalías asociadas, especialmente un secuestro pulmonar. En el período postnatal, nuestros pacientes fueron estudiados predominantemente 
con Tomografía Computada (TC) de tórax lo cual es concordante con lo relatado en la literatura en que se señala la importancia de esta técnica en la caracterización de la lesión en el período postnatal $^{2}$. En 2 pacientes se realizó Angio-TC que descartó la presencia de un secuestro pulmonar asociado. Se destaca que en esta serie existió una alta correlación entre los hallazgos de los estudios de imágenes y los hallazgos anatomopatológicos. En un recién nacido con distrés respiratorio severo se llegó al diagnóstico sólo con radiografía de tórax, por la necesidad de operar oportunamente. La comorbilidad asociada fue baja objetivándose en sólo dos pacientes (un paciente con pectus excavatum y un paciente con comunicación interventricular), lo cual concuerda con otras series publicadas $^{21}$.

El tipo de cirugía elegida fue la lobectomía del sitio afectado tal como se ha señalado en la literatura $^{22,23}$. El método quirúrgico fue la toracotomía abierta. A pesar de esto, existen publicaciones recientes que describen a la videotoracoscopia como una alternativa a la cirugía convencional ya que disminuye la estadía hospitalaria, los días con tubo de drenaje y las complicaciones postoperatorias $^{24-26}$, pero esta técnica no siempre está disponible, ya que, se necesita de mayor implementación que la cirugía tradicional.

La evolución de los pacientes en general fue buena, sólo se presentaron complicaciones menores como atelectasias lobares que respondieron a manejo con kinesiterapia respiratoria al cabo de 2 días. No registramos mortalidad a diferencia de lo registrado en otras series de casos clínicos ${ }^{10}$ ni complicaciones mayores como neumotórax a tensión ${ }^{14}$ debido probablemente al alto porcentaje de diagnóstico prenatal, a la sospecha diagnóstica precoz y al manejo con un equipo multidisciplinario. Ninguno de los pacientes ha presentado escoliosis. La estadía hospitalaria tuvo una mediana de 5,5 días similar valor a lo referido por la literatura nacional y extranjera ${ }^{14,24}$.

La presentación de esta serie de casos clínicos traduce la experiencia de nuestro centro asistencial en los últimos años, enfatizándose la importancia de la sospecha diagnóstica adecuada y el manejo multidisciplinario de esta patología.

\section{Conclusiones}

Se presenta la serie clínica de malformación congénita de la vía aérea pulmonar o malformación quística adenomatoidea congénita del Hospital Roberto del Río entre los años 2000 y 2014 enfatizando la importancia de la detección precoz y su buena evolución clínica concordante con lo señalado en la literatura.

\section{Bibliografía}

1.- STOCKER J T. Congenital pulmonary airway malformation-a new name for an expanded classification of congenital cystic adenomatoid malformation of the lung. Histopathology 2002; 41 (Suppl 2): 424-58.

2.- KOTECHA S, BARBATO A, BUSH A, CLAUS F, DAVENPORT M, DELACOURT C, et al. Antenatal and postnatal management of congenital cystic adenomatoid malformation. Paediatr Respir Rev 2012; 13: 162-70.

3.- EUROCAT: European Surveillance of Congenital Anomalies Disponible en: www.eurocat-network.eu [última revisión el 22 de marzo de 2015].

4.- STOCKER J T. Cystic lung disease in infants and children. Fetal Pediatr Pathol 2009; 28: 155-84.

5.- LANGSTON C. New concepts in the pathology of congenital lung malformations. Sem Pediatr Surg 2003; 12: 17-37.

6- RAYCHAUDHURI P, PASUPATI A, JAMES A, WHITEHEAD B, KUMAR R. Prospective study of antenatally diagnosed congenital cystic adenomatoid malformations. Pediatr Surg Int 2011; 27: 1159-64.

7.- PATELLA M, ANILE M, DISO D, ONORATI I, VENUTA F. Unusual presentation of congenital cystic adenomatoid malformation of the lung. Lung 2012; 190 : 697-8.

8.- PULIGANDLA P S, LABERGE J M. Congenital lung lesions. Clin Perinatol 2012; 39: 331-47.

9.- GUIDRY C, MCGAHREN E D. Pediatric chest I: Developmental and physiologic conditions for the surgeon. Surg Clin North Am 2012; 92: 615-43.

10.- GIUBERGIA V, BARRENECHEA M, SIMINOVICH M, PENA H G, MURTAGH P. Congenital cystic adenomatoid malformation: clinical features, pathological concepts and management in 172 cases. J Pediatr (Rio J) 2012; 88: 143-8.

11.- FERREIRA H P C, FISCHER G B, FELICETTI J C, CAMARGO J J P, ANDRADE C F. Surgical treatment of congenital lung malformations in pediatric patients. J Bras Pneumol 2010; 36: 175-80.

12.- HERMAN T E, SIEGEL M J. Type 2 CPAM with delayed-onset Bochdalek hernia. J Perinatol 2012; 32 : 309-11.

13.- DEBOER E M, KEENE S, WINKLER A M, SHEHATA B M. Identical twins with lethal congenital pulmonary airway malformation type 0 (acinar dysplasia): further evidence of familial tendency. Fetal Pediatr Pathol 2012; 31: 217-24.

14.- ZÚÑIGA S, SOTO G, SEPÚLVEDA C, GARCÍA C, CONTRERAS O, SÁNCHEZ I. Una década de experiencia en malformación adenomatoidea quística del pulmón. Enferm Respir Cir Torac 1998; 14: 128-36.

15.- ÁlVAREZ C, CERDA C, CERDA C, SANHUEZA B. 
Malformación congénita de la vía aérea pulmonar: reporte de un caso adulto. Rev Chil Enferm Respir 2009; 25: $182-7$.

16.- ESCOBAR F, FUENTES F, URZÚA C, CAPETILLO M. Malformación adenomatoidea quística pulmonar de presentación tardía: revisión del tema y presentación de un caso. Rev Chil Radiol 2010; 16: 190-4.

17.- DAVENPORT M, EBER E. Long term respiratory outcomes of congenital thoracic malformations. Semin Fetal Neonatal Med 2012; 17: 99-104.

18.- RECIO RODRÍGUEZ M, MARTÍNEZ DE VEGA V, CANO ALONSO R, CARRASCO ARRANZ J, MARTÍNEZ TEN P, PÉREZ PEDREGOSA J. MR imaging of thoracic abnormalities in the fetus. Radiographics 2012; 32: E305-21.

19.- AKSOY OZCAN U, ALTUN E, ABBASOGLU L. Space occupying lesions in the fetal chest evaluated by MRI. Iran J Radiol 2012; 9: 122-9.

20.- SHEARES B. Recurrent pneumonia in children. Pediatr Ann 2002; 31: 109-14.

21.- ANTÓN-MARTÍN P, CUESTA-RUBIO M T, LÓPEZGONZÁLEZ M F, ORTIZ-MOVILLA R, LORENTE-
JAREÑO M L, LÓPEZ-RODRÍGUEZ E, et al. Malformación adenomatoidea quística congénita. Rev Chil Pediatr 2011; 82: 129-36.

22.- MULLER C O, BERREBI D, KHENICHE A, BONNARD A. Is radical lobectomy required in congenital cystic adenomatoid malformation? J Pediatr Surg 2012; 47: 642-5.

23.- BAIRD R, PULIGANDLA P, LABERGE J M. Congenital lung malformations: Informing best practice. Sem Pediatr Surg 2014; 23: 270-7.

24.- VU L T, FARMER D L, NOBUHARA K, MINIATI D, LEE H. Thoracoscopic versus open resection for congenital cystic adenomatoid malformations of the lung. $\mathrm{J}$ Pediatr Surg 2008; 43: 35-9.

25.- NASR A, BASS J. Thoracoscopic $v s$ open resection of congenital lung lesions: a meta-analysis. J Pediatr Surg 2012; 47: 857-61.

26.- SHIMIZU S, YAMASHITA Y, MUKAIDA H, HIRABAYASHI N, EGAWA H, KANEKO M, et al. Thoracoscopic resection of congenital cystic adenomatoid malformation in an adolescent. Asian J Endosc Surg 2013; 6: 44-7.

Correspondencia a:

Dr. Guillermo Zepeda F.

Departamento de Pediatría y Cirugía Infantil,

Facultad de Medicina Universidad de Chile.

Hospital Roberto del Río. Santiago de Chile.

Email: gezepeda@med.uchile.cl 